\title{
Importancia y evolución del concepto de conciencia del tiempo en la fenomenología de Edmund Husserl
}

\author{
Importance and evolution of the concept \\ of time-consciousness in \\ Edmund Husserl's phenomenology
}

FRANCISCO CONDE SOTO

Recibido: 19-12-2008 Aprobado definitivamente: 02-02-2009

\section{RESUMEN}

Este estudio ofrece una presentación del trabajo de investigación desarrollado en una tesis doctoral sobre el problema de la conciencia del tiempo en la fenomenología de Husserl defendida en la Universidad de Barcelona en abril del 2007. Tras justificar la importancia que el tema tiene para el pensamiento de Husserl son presentados los diferentes acercamientos a esta cuestión en cada una de las épocas de su pensamiento, distinguiendo fundamentalmente tres periodos: las lecciones sobre la conciencia interna del tiempo (1904/05), los manuscritos de Bernau (1917/18) y los manuscritos C (1929/34).

\section{PALABRAS CLAVE}

FENOMENOLOGÍA, TIEMPO, CONCIENCIA, HUSSERL.

\section{ABSTRACT}

This paper offers a general overview of a research developed and defended as a $\mathrm{PhD}$ at the University of Barcelona in April 2007 concerning the problem of time- consciousness in Husserl's phenomenology. After justifying the relevance of the subject to Husserl, we present the different approaches to the problem of time-consciousness in every period of his thinking, distinguishing mainly three phases: the lessons about inner time consciousness (1904/05), the Bernauer manuscripts (1917/18) and the C- manuscripts (1929/34).

\section{KEYWORDS}

PHENOMENOLOGY, TIME, CONSCIOUSNESS, HUSSERL. 


\section{LA IMPORTANCIA DEL TEMA DEL TIEMPO EN LA FENOMENOLOGÍA DE HUSSERL}

El ESTUdio DE LA TEMPORALIDAD no es algo casual en el pensamiento de Husserl. Lejos de motivarse en la curiosidad intelectual, la aclaración de en qué consiste la conciencia del tiempo puede ser considerada una de las claves de la fenomenología. Husserl no se acerca al problema de la temporalidad en abstracto para formular una teoría acerca de si el tiempo es cíclico o no, o acerca de en qué consista la eternidad, el sentido de la historia, etc. Tampoco se trata de una teoría psicológica en torno a la percepción subjetiva del tiempo: es decir, no se trata de un intento por explicar por qué en ocasiones el tiempo pasa más o menos deprisa, explicando nuestras sensaciones subjetivas, psicológicas de tiempo. Ni tampoco es un intento de explicación de la relevancia de la finitud o infinitud temporal de mi existencia.

El tiempo es ante todo uno de los aspectos esenciales de la constitución de cualquier objeto. Todo objeto es una unidad dotada de una determinada duración que debe ser constituida por la conciencia. La conciencia del tiempo o conciencia interna es el movimiento de constitución primero sobre el que son posibles todos los otros; constituye el sistema de puntos objetivos temporales en el que vienen a encuadrarse los diferentes datos de sensación. Que entre diferentes daciones puntuales se establezcan relaciones de unidad es posible en virtud de la fijación de la identidad de cada una de estas daciones y en virtud de la relación entre varias de estas identidades. El tiempo es principio de individuación para los contenidos dados en él: a partir del momento en que un contenido se da, lo hace en una posición temporal que le corresponderá unívocamente ya para siempre, sin perjuicio de que un contenido similar vuelva a aparecerse en posteriores ocasiones.

Husserl lleva a cabo en la primera época de su pensamiento un estudio de la intencionalidad de los diferentes actos constitutivos (percepción, recuerdo, fantasía, conciencia de imagen). Cada uno de los actos de conciencia es comprendido como una cierta aprehensión animadora animación intencional de una serie de datos de sensación que en virtud de aquella se convierten en presentadores de los distintos aspectos de un objeto y del objeto mismo en cuanto unidad intencional apuntada por todos ellos: «[...] en el acto del aparecer es vivida la complexión de sensación, en cierto modo 'aprehendida', 'apercibida', y en este carácter fenomenológico de la aprehensión animadora efectuada sobre las sensaciones consiste eso que llamamos el aparecerse del objeto» (Hu XIX/1, $5^{\text {a }}$ Investigación Lógica, 360¹).

1 Las citas de las obras de Husserl se hacen según el procedimiento canónico precisando el volumen de la edición crítica en alemán (Husserliana) y la página. Véanse las referencias bibliográficas al final del texto. 
La comprensión de estas diferentes intencionalidades se hace en primera instancia desde un punto de vista fundamentalmente estático: son supuestas una aprehensión y una serie de datos de sensación aprehendidos en el mismo momento temporal que el objeto que se aparece. Tanto los datos de sensación como la aprehensión a ellos referida son a su vez unidades que duran y cuya constitución debe ser estudiada por la fenomenología del tiempo.

El punto de partida es entonces el estudio acerca de cómo es posible la percepción de objetos que duran, objetos que tienen que ser el resultado de alguna peculiar síntesis entre elementos puramente presentes y puramente pasados. El análisis comienza con un estudio de las condiciones de posibilidad de la percepción de los objetos temporales, de los objetos dotados de duración, escogiendo como ejemplo paradigmático una melodía: es necesario explicar cuáles son las estructuras mediante las que es posible que una serie de elementos pasados se vinculen a otros presentes y sean percibidos como formando una unidad con ellos. El desarrollo de esta cuestión, que es la que estrictamente merece el rótulo de conciencia originaria del tiempo, conduce a un análisis de la estructura de la conciencia constituidora de tal síntesis.

\section{LA FENOMENOLOGÍA DEL TIEMPO DESDE LAS LECCIONES DEL SEMESTRE DE IN- VIERNO DE 1904/05 (Hu X) HASTA IDEAS I (Hu III)}

En el semestre de invierno de 1904/05 Husserl imparte una lección titulada «Problemas fundamentales del conocimiento» cuyas tres primeras partes estaban dedicadas respectivamente a la percepción, a la atención y a la distinción entre los actos de fantasía y los actos de conciencia de imagen. En la cuarta parte se desarrolla el primer acercamiento explícito de Husserl al problema de la percepción de un objeto que dura. El punto de arranque es la necesidad de una desconexión o puesta fuera de juego [Ausschaltung] del tiempo objetivo, es decir, de la idea de que hay un tiempo natural-objetivo-lineal, remitiéndose así a un análisis de los datos inmanentes de la conciencia.

En Investigaciones lógicas Husserl había trabajado bajo el presupuesto de que tanto los contenidos de sensación pre-intencionales como las aprehensiones son simultáneas, de forma que parecía que se trataba de elementos situados fuera del tiempo, es decir, no había ningún cuestionamiento acerca de su génesis temporal. En la quinta y la sexta investigaciones la percepción era concebida como formada por un acto de aprehensión de un determinado contenido de sensación mediante la que se aparecía el objeto percibido. El contenido de sensación aprehendido se corresponde a la parte del objeto que es propiamente percibida (Hu XIX/2, 6 a Investigación Lógica, §22) junto a una presentación no intuitiva de aquellas partes del objeto que en este momento no están siendo percibidas. Este mismo modelo es empleado por Husserl en las lecciones de 1904 para determinar que la percepción de la unidad temporal de 
un objeto que dura se forma a partir de una impresión originaria intuitiva y una serie de retenciones y protenciones no intuitivas. Husserl aplica el modelo de la percepción espacial también a la constitución del tiempo objetivo para afirmar que la percepción de un objeto que dura es posible en virtud de la duración del contenido de sensación aprehendido. La cuestión es entonces explicar como es posible la síntesis entre los diferentes momentos de ese contenido: los pasados se unen a la impresión dadora del contenido actual al ser conservados en el presente mediante un recuerdo reciente que no puede ser considerado ningún tipo de presentificación [Vergegenwärtigung] de la fantasía o de conciencia de imagen. La primera solución propuesta consiste en comprender esta retención como un contenido de sensación de igual talante que los contenidos dados impresionalmente en el ahora. Una diferente aprehensión es la responsable de que ellos entrasen en la percepción del objeto que dura como fases inmediatamente pasadas del objeto que dura. Los intentos para aclarar de qué tipo es esta aprehensión pasarían por su comparación con los actos de conciencia de imagen, de fantasía y de rememoración.

¿Cómo saber que las diferentes apariciones de un mismo objeto pertenecen al mismo objeto o que una serie de sonidos singulares pertenecen a una misma melodía? La fenomenología del tiempo tiene como primer objetivo explicar la combinación de fases pasadas, presentes y futuras que se unen para que podamos percibir la unidad de un objeto que dura.

Para ello Husserl introduce una estructura formada por tres elementos: protoimpresión, retención y protención, que son las partes del acto perceptivo que se refieren a las fases presente, pasada y a la fase futura que va a llegar de forma inminente. De la protención no se ocupa en este momento.

Husserl afirma que cada intuición momentánea es la responsable de la dación de una fase actual del objeto: una fase que está dotada del carácter del «ahora», de la posición de algo «como sí mismo» [als Selbst] en el momento presente. La protoimpresión es el «impresionar» en la conciencia que aporta de forma continua un nuevo contenido dado bajo la forma temporal de ahora. Más que de actos de impresión aislados de lo que se trata es de un constante entrar en la esfera de la conciencia nuevos contenidos originarios. El caso paradigmático de objeto que dura es una melodía formada por varios sonidos. A cada percepción del sonido intuido ahora se le une un recuerdo primario del sonido anterior de forma que sería posible captar la sucesión que forman los dos. El surgimiento de contenidos correspondientes a los ahoras que se suceden unos a otros no es el resultado de un proceso de constitución anterior, sino que las impresiones originarias nacen y se suceden unas a otras de por sí. Una vez dada, esta conciencia del contenido del punto de ahora se modifica haciéndose cada vez más y más pasada, y convirtiéndose en retención. 
El principal problema que le plantea esta retención o recuerdo reciente a Husserl es que no puede ser clasificado en ninguno de los tipos de actos intencionales que hasta aquel entonces había reconocido: esa parte del acto perceptivo que nos da una fase pasada no es ni una percepción, ni una conciencia de imagen ni un acto de fantasía. Su conclusión es que la retención o conciencia primaria de pasado es un acto de conciencia tan peculiar y particular como lo puede ser la conciencia originaria de un contenido dado en el punto de ahora. El contenido de un recuerdo reciente no es una «modificación» de un contenido primario que ya no es un contenido primario, sino precisamente una modificación, «algo totalmente diferente», «conciencia de sensación pasada» (Hu X, 324).

La cuestión es que esta insistencia en el carácter peculiar de presentación y recuerdo reciente termina por volver a plantear el problema de saber qué relación existe entre el momento presente y este mismo momento retenido, sólo que a otro nivel. Si retención, protención e intuición de un ahora puntual son modos igualmente originarios que no pueden ser explicados o derivados unos de otros, parece que finalmente no se consigue explicar como es posible la conservación del contenido de un ahora, o lo que es lo mismo, como es posible la conciencia originaria del tiempo.

El otro rasgo que caracteriza a esta primera fenomenología del tiempo de la primera época es su no asunción de un tiempo objetivo-lineal: no se puede contar con la suposición ingenua de que existe una corriente de vivencias formada por todas y cada una de las vivencias singulares ordenadas en una línea que sería el tiempo objetivo. Los datos con los que se cuenta son los datos de la conciencia (inmanente) y es sobre ellos como será posible explicar la constitución del tiempo objetivo. Si en un primer momento el propio Husserl considera como equivalentes el tiempo de la conciencia y el tiempo inmanente/objetivo, enseguida rectifica su postura. Sólo cuando se objetiva el sentir el inicio del sonido de una campana podemos hablar de una sensación que vendría a coincidir temporalmente con el inicio del sonido objetivo externo de la campana. Cuando una sensación es objetivada se convierte en una dación del tiempo objetivado y es entonces cuando se puede hablar de un tiempo en la conciencia. Antes de este sólo hay un cuasi-tiempo vivido. Cuando escuchamos una melodía sin reflexionar sobre esta percepción advertimos que discurre sin que identifiquemos que un sonido va delante de otro. En el fluir hay unidades de forma previa a la objetivación, pero no se trata de unidades propiamente temporales. El tiempo es propiamente una forma referente a las objetivaciones efectuadas sobre el plano de las vivencias, en la medida en que sólo tras estas objetivaciones es posible identificar una vivencia como formada por una serie de fases que se suceden. Cuando vivimos arrojados a la percepción esta transcurre como si no hubiese tiempo; es cuando reflexionamos sobre ella, advertimos que había un inicio, un transcurso y un fin situados en un tiempo que Husserl denomina «inmanente». Tan sólo gracias a la 
objetivación de las vivencias de la conciencia surge un tiempo, surgen objetos individuales dotados de una posición temporal. El tiempo es la forma posible de toda objetivación individual, de toda aparición: pero esto no implica que las vivencias de la conciencia estén ya ordenadas en un tiempo.

En sus lecciones del semestre de invierno 1906/07, tituladas «Introducción a la lógica y a la teoría del conocimiento». Se trata del III. Apartado, titulado «Las formas de objetivación» (Hu XXIV, capítulo 7, 243-274). Estos textos resultan especialmente relevantes porque en ellos se habla por primera vez de la «conciencia absoluta» del tiempo y su relación con la conciencia inmanente. Los objetos inmanentes resultarían de algún tipo de aprehensión de una serie de componentes ingredientes efectuada por una determinada conciencia absoluta. La novedad es que estos componentes ingredientes, componentes de sensación, son pensados como el resultado de algún tipo de constitución intencional: ser sentido no equivale ya a ser el mero contenido ingrediente de una vivencia de sensación, sino que es necesario un cuestionamiento acerca del origen de los datos de sensación. ${ }^{2}$

En los textos complementarios del cuarto apartado del tomo X de Husserliana, redactados entre 1907-09 se propone por primera vez un cierto abandono del modelo acto de aprehensión/contenido de aprehensión para explicar el peculiar tipo de intencionalidad que opera en la conciencia del tiempo. Husserl afirma ahora que en el caso de la conciencia de tiempo se hace necesario prescindir por completo de este esquema y suponer que son posibles constituciones que no lo respetan. Los temas de mayor novedad son la teoría de una doble intencionalidad del flujo absoluto de tiempo (transversal y longitudinal), así como la aclaración de que la aprehensión temporal, la intencionalidad temporal, no es un tipo de aprehensión objetiva. Por lo que respecta al segundo punto Husserl efectúa un cambio fundamental en la concepción del recuerdo primario, que ahora pasará a ser considerado una modificación de un tipo peculiar y en todo caso ninguna aprehensión de un contenido presente a través de la que se apuntaría a un objeto no presente. La conciencia del tiempo es posible gracias a una serie de «adumbraciones» o «perfilaciones» [Abschattungen] de este objeto que no son propiamente percibidas, pero de las que se tiene alguna conciencia. La conciencia o vivencia de estas peculiares «adumbraciones» será denominada a partir del año 1906/07 «conciencia absoluta». No se trata de ningún tipo de conciencia de objeto, sino que es una «conciencia absoluta» de los objetos temporales inmanentes que a su vez se percibe a sí misma como cierto ordenamiento cuasi-temporal pre-objetivo. Esta conciencia absoluta, que sería conciencia de la ejecución de los modos de impresión, retención y protención

2 En la traducción del término «reell» seguimos lo apuntado por García-Baró (1993, p. 263). 
no sería una conciencia intencional. Ya no se podría seguir hablando de una serie de contenidos ingredientes a la conciencia, sino que estos se mostrarían ahora como el resultado de un proceso dador previo. La impresión originaria es un sentir un contenido, una sensación, pero no se trata de ningún contenido estático; se trata principalmente de un modo de estar en la conciencia, no de algo que está en la conciencia; la retención es así mismo de forma directa una conciencia del carácter de pasado de un contenido sin que tenga que haber ningún contenido ingrediente-presente que represente a aquel. Por otra parte, la conciencia absoluta y cada una de sus vivencias se mostrarían ahora como siendo intemporales, puesto que no habría propiamente hablando contenidos ingredientes en ellas que pudiesen ser situados en una determinada posición dentro de una sucesión temporal: la impresión, la retención y la protección «captan» el darse, haberse dado y estar-a-punto de darse de un contenido, pero no se trata de ningún acto o duración. Otro de los descubrimientos de esta época sería la postulación de una doble dirección intencional de la retención que permitiría que la conciencia constituyese de forma pre-reflexiva su propio movimiento de temporalización. La conciencia absoluta constituye el flujo intemporal de la conciencia absoluta mediante una «intencionalidad longitudinal»-el remitir de una retención a las anteriores-y la sucesión de los objetos temporales mediante una «intencionalidad transversal». Además, el flujo se aparece a sí mismo bajo la forma de un flujo.

El problema del tiempo lleva a Husserl a renunciar al esquema aprehensión/ contenidos de aprehensión.

a) En primer lugar, porque la parte del acto perceptivo que es la retención no puede ser explicada como una aprehensión de contenidos: los contenidos serían pasados y la aprehensión presente.

b) En segundo lugar: porque los propios contenidos de aprehensión son el resultado de una génesis temporal. El flujo de conciencia no está formado por datos entendidos «como objetos por así decir terminados» [als sozusagen fertigen Gegenständen] (Hu XVII, 291). Si ni los datos de sensación ni los actos de aprehensión son objetos ya terminados, listos es porque hay un proceso previo a través del que surge su unidad temporal, el proceso de síntesis a través del que se forma su unidad. Dato hilético pasa por tanto a significar cada una de las unidades momentáneas dadas en el protoproceso.

c) En tercer lugar, porque en la vida de conciencia no se dan los datos sensibles y las aprehensiones intencionales por separado.

d) Pero finalmente, no se trata tan solo de que la retención no pueda ser explicada como una aprehensión de unos contenidos ingredientes, sino que incluso aquellos núcleos hiléticos dados por la protopresentación no son elementos ingredientes que se ofrecen a una aprehensión: Husserl cambia su concepto de una conciencia estática por una conciencia dinámica. Los datos de sensación son 
sentir, presentan de alguna forma directamente, sin necesidad de la mediación de una aprehensión, de forma que el significado del término «ingrediencia» se refiere precisamente a esta su función de dación originaria.

La conciencia de la duración de un objeto es posible gracias a una serie de retenciones o «adumbraciones» [Abschattungen] de este objeto que no son propiamente percibidas, pero de las que se tiene alguna conciencia, conciencia que es denominada a partir del año 1906/07 «conciencia absoluta». No se trata de ningún tipo de conciencia de objeto, ninguna conciencia intencional, sino una «conciencia absoluta» de los objetos temporales inmanentes: cierto ordenamiento cuasi-temporal pre-objetivo, conciencia o estarse ejecutando de la impresión, la retención y la protención.

La impresión originaria es un sentir un contenido, una sensación, pero no ningún contenido estático; se trata de un modo de estar en la conciencia, no de algo que está en la conciencia; la retención es así mismo de forma directa una conciencia del carácter de pasado de un contenido sin que tenga que haber ningún contenido ingrediente-presente que represente a aquel. Por otra parte, la conciencia absoluta y cada una de sus vivencias son intemporales: no contienen contenidos ingredientes situables en una determinada posición dentro de una sucesión temporal: la impresión, la retención y la protención «captan»el darse, haberse dado y estar-a-punto de darse de un contenido, pero no se trata de ningún acto, sino de partes de un acto.

Husserl está descubriendo la necesidad de hablar de una conciencia absoluta situada más allá del plano inmanente. La conciencia absoluta en sí misma no es nada temporal. Sólo percibimos la temporalidad de nuestras vivencias cuando reflexionamos sobre ellas, cuando se les impone una forma temporal que las coloca e introduce en el tiempo secuencial, es decir, en la corriente o sucesión inmanente. La vivencia en cuanto conciencia interna no es ningún tipo de duración, aunque se pueda afirmar ya que se trata de algún tipo de «unidad» configurada mediante un decurrir o fluir característicos.

Tenemos por tanto un flujo absoluto consistente en vivencias en el sentido de vivir. Lo importante a nivel temporal es que aunque se trate de un flujo continuo de «cambio» no hay en realidad ningún cambio, porque no hay ningún objeto que cambie. La protoimpresión y el continuo de retenciones que se le adhieren no son nada en el tiempo inmanente, sino momentos del flujo. Nada temporal, sino el movimiento previo a partir del que surge el tiempo.

Las dificultades que introduce el concepto de conciencia interna en el texto de Husserl son numerosas. Nuestra interpretación es que la conciencia absoluta en el sentido de vivencias meramente vividas, la conciencia interna del tiempo y la conciencia interna son diferentes perspectivas bajo las que puede ser contemplada una única conciencia absoluta. El término «percepción o conciencia interna» no se refiere a un acto de percepción habitual, sino al mero vivir sus 
vivencias por parte de la conciencia. La elección del término «interna» respondería al hecho de que se trata del tipo de conciencia que la conciencia tiene «interiormente» de sus vivencias.

Por otra parte, la conciencia tiene que guardar consigo mismo algún tipo de relación de constitución, si no se quiere recurrir a infinitos planos de conciencia: Husserl formula la teoría de una doble intencionalidad (transversal y longitudinal). La conciencia absoluta constituye su propio fluir mediante una «intencionalidad longitudinal»-el remitir de una retención a las anteriores- y constituye la sucesión de los objetos situados en el tiempo mediante una «intencionalidad transversal». Es decir, el flujo se autoaparece a sí mismo bajo la forma de un flujo. Este hecho permite explicar el funcionamiento de la conciencia sin recurrir a un plano de conciencia posterior porque cada retención capta aquello que la precede como algo que la precede: se explica así la posibilidad de captar el movimiento mediante el que una retención retiene otra anterior, o lo que es lo mismo, el movimiento mediante el que una fase de la conciencia sucede a otra. De esta forma el flujo «percibe» (entre comillas) el sucederse de estas fases en función de la cadena generada por las retenciones. El propio movimiento del flujo consiste en el hecho de que cada nueva fase recoge a la anterior y se capta a sí misma como «sucediendo» a aquella dentro de una misma continuidad.

\section{Los MANUSCRItos De BeRnaU ACERCA DE LA} CONCIENCIA DEL TIEMPO (1917-18)

El siguiente momento de la fenomenología del tiempo lo constituyen los manuscritos de Bernau acerca de la conciencia del tiempo, redactados en los años 1917 y 1918 (Hu XXXIII). El tema más importante de estos manuscritos es aclarar si la conciencia es una conciencia intencional tempoconstituidora en todo momento y por sí misma o si por el contrario sus objetos son captados como el resultado de una constitución intencional únicamente cuando el yo se dirige atentamente a ellos, de forma que él activaría así una serie de síntesis previas no intencionales.

La primera alternativa considera la conciencia de tiempo como una conciencia que no constituiría propiamente hablando objetos, sino que se mostraría con este carácter intencional-constituidor únicamente cuando los objetos fuesen término de una captación propia efectuada por el yo: no se podría hablar de intencionalidad antes de que el yo se dirigiese atentamente a uno de los objetos constituidos. De por sí, la conciencia originaria del tiempo consistiría en un mero flujo de datos primarios no tempoconstituidor. Este primer modelo implicaría una modificación en la teoría de la atención de Husserl, consistente en la defensa de que la atención no es algo que venga meramente a recubrir un proceso intencional de constitución anterior, sino que la atención a este puede ahora transformar algo no intencional en intencional. Entre otros problemas, uno 
de los que acosaría a este modelo sería que no es comprensible como un mero flujo de datos de conciencia puede ser transformado a través de la atención en un flujo constituidor de unidades temporales.

El segundo modelo de los manuscritos de Bernau postula una conciencia de tiempo que constituye continuamente unidades temporales y que precede a las captaciones atentas por parte del yo efectuadas sobre ella: la atención simplemente vendría a recorrer la corriente de conciencia y las unidades formadas con anterioridad en esta sin efectuar más transformaciones que aquellas relacionadas con un cambio en el modo atencional.

En lo que se refiere estrictamente a la conciencia interna del tiempo se descubre que tanto las retenciones como las protenciones se unen a las protoimpresiones mediante un proceso de asociación originaria pasiva previa a toda intervención del yo. La perspectiva temporal es reinterpretada aquí como una perspectiva afectiva, de forma que la retención sería considerada una pérdida progresiva de la capacidad de afectar distintamente de los diferentes elementos de un contenido. Por otra parte, la rememoración y la expectativa pueden ser comprendidas como un cierto despertar asociativo consistente en la transmisión de una determinada fuerza afectiva desde un elemento presente a otro pasado o a otro futuro sobre el que se genera cierta «representación».

En los manuscritos de Bernau nace además lo que vendrá a llamarse el análisis genético o análisis de la síntesis pasiva. Ciertos rasgos de la concepción del tiempo de los manuscritos de Bernau están en sintonía con la doctrina de la síntesis pasiva desarrollada en el tomo XI de Husserliana. Por ejemplo, la influencia que ahora se le reconoce al pasado y a las retenciones correspondientes sobre la forma determinada que adquiere una protención, la existencia de una serie de «habitualidades»; la introducción del concepto de afección y la puesta en juego del plano egoico, como el elemento afectado por ellas. El concepto de asociación viene a cobrar significado en el contexto de la formación de las diferentes unidades hiléticas materiales pre-afectivas. La síntesis pasiva en este ámbito se refiere a la necesidad de que antes de toda intervención de un yo diferentes unidades hiléticas se destaquen de un determinado fondo sensible estableciendo entre ellas diferentes unidades que vienen a ejercer una tendencia a ser captadas sobre el yo. A su vez los objetos formados por estas unidades hiléticas podrían asociarse entre sí de forma pasiva y dar lugar a la configuración de unidades de mayor complejidad, también antes de que el yo se dirigiese atentamente a ellas y las captase. La distinción entre forma y materia, que hasta ahora venía siendo interpretada como una distinción entre un acto de aprehensión y un contenido de aprehensión, es sustituida por la distinción entre un campo yoico y un campo no-yoico. La conciencia era interpretada como el lugar donde se darían una serie de datos de sensación y un acto de aprehensión que los captaría. Husserl hace «retroceder» esta distinción y la sitúa en el terreno genético: los datos de 
sensación no son unos meros datos que aparecen en la conciencia sin más, sino que proceden de una constitución anterior que se produce de forma pasiva antes de toda captación (activa) por parte del yo. Esto implica una cierta superación de la noción de unos contenidos de sensación inertes, aintencionales, necesitados de una aprehensión para ganar sentido y ejercer su función presentadora. Los datos hiléticos establecen relaciones entre sí en virtud de ciertas remisiones intencionales de unos a otros, de forma que antes de ser propiamente aprehendidos podrían constituir ya unidades temporales.

La idea principal que subyace a los textos acerca de la síntesis pasiva es que la conciencia se encuentra en todo momento en contacto con una serie de datos hiléticos dotadas de un determinado índice de fuerza afectiva que son el resultado de una constitución pasiva previa. Algunas de estas unidades son posteriormente objeto de actos de atención explícita por parte del yo y pasan a ejercer afecciones propiamente dichas. Parece entonces que antes de la captación atenta efectuada por el yo hay que hablar de algún tipo de presencia a la conciencia de los destacamientos [Abhebungen] de fuerzas afectivas. Dicho de otra forma es necesario preguntarse para quién se dan las pre-afecciones previas, porque aunque se hable de un «no tomar parte inactivo» tiene que tratarse de algún modo de presencia de algún tipo de polo egoico. En este sentido es posible afirmar que la esfera de la subjetividad trascendental tiene que ir más allá de la esfera del yo trascendental: incluiría también un cierto proto-yo o vida protofluyente a los que corresponden una serie de procesos de constitución pasivos.

El proceso constitutivo es el siguiente: a) una constitución del tiempo de la que surge la unidad de cada dato hilético gracias fundamentalmente a las síntesis de cubrimiento entre las retenciones referentes a un mismo dato hilético; b) el establecimiento de relaciones asociativas entre estos datos para formar objetos; que a su vez establecen entre ellos relaciones asociativas de un nivel superior. Los datos hiléticos no son ciertamente nada más que un cierto preser que aguarda determinada síntesis de cubrimiento retencional para entrar en el plano del ser-temporal-inmanente. Se trata de cierta «materia» o cierta «multiplicidad» anterior.

Uno de los errores que deben ser evitados es considerar que hay una síntesis temporal que precede a una síntesis asociativa, de forma que la conciencia de tiempo y la síntesis pasiva se relacionen como una forma y un contenido. Los procesos de la conciencia de tiempo no se producen de forma separada a los procesos de constitución pasiva-hilética, sino que ambos elementos están operando continuamente de forma conjunta, independientemente de que en el análisis puedan ser captados abstractivamente como algo separado.

El problema es que nuestro acceso al campo hilético es fenomenológicamente no evidente. Todas las afirmaciones acerca del campo pasivo no pueden superar su estatus de suposiciones. Depraz califica directamente esta fenome- 
nología como una «fenomenología metafísica», porque el estudio de la hyle es el estudio de aquello que aunque permite cualquier aparición intencional, ello mismo no se aparece. La hyle, antes que referirse a las sensaciones constituidas, se refiere al campo de la pre-asociación genética (Depraz 2000). Tras la reducción, los datos hiléticos y la síntesis pasiva que se produce entre ellos se convierten en un factor trascendente a la conciencia pura, contenidos cuyo origen es algo extraño, algo diferente a la conciencia que esta no puede justificar. De esta forma la constitución termina por depender de un hecho que es ajeno al yo, una serie de datos no-egoicos que no resultan de ninguna operación del sujeto (Walton 1993, p. 42). El concepto de intencionalidad pasiva viene a recoger esta ambiguiedad.

La crítica especializada insiste en la problematicidad del concepto de síntesis pasiva y de las dificultades para concebir como las fuerzas afectivas pre-objetivas se relacionan con el yo antes de que este se dirija a ellas. ${ }^{3} \mathrm{Si}$ la presencia del yo en la síntesis pasiva consiste en un «no tomar parte inactivo» se trata ya de algún modo de presencia del yo.

La oposición actividad - pasividad no consiste en la oposición entre formamateria o entre algo propio de la conciencia y algo ajeno a ella. En los años 30 Husserl se replantea la posibilidad de poder hablar de una síntesis pasiva previa al yo: también a la síntesis pasiva la acompaña un yo de los actos y de las habitualidades que está aquí en desarrollo. Las vivencias pasivas no son algo egoico, pero se encuentran «para el yo» ahí: en la medida en que representan una tendencia a despertar la captación por parte del yo, están centradas en torno a alguna forma de presencia egoica, concretamente, a un proto-yo, concepto central en los manuscritos $\mathrm{C}$.

\section{El PROBLEMA DEL TIEMPO EN LOS MANUSCRITOS C (1929/34)}

El último apartado de este estudio se ocupa de los desarrollos que sufre la conciencia del tiempo en los manuscritos C. Fragmentarios y aporéticos han sido editados en el año 2006 por el director del archivo Husserl de Colonia, Dieter Lohmar (Hu - Manuscritos C). Se trata, al igual que los manuscritos de Bernau, de un grupo de manuscritos de investigación que, entre otros numerosos temas, siguen y desarrollan la línea del análisis genético. Su tema principal es la identificación de la conciencia absoluta con una subjetividad transcendental-

3 «Los manuscritos publicados hasta el momento no permiten una determinación sistemática de la síntesis pasiva, porque Husserl nunca se expresó de forma unívoca acerca del estatus de esta forma de constitución. No en último lugar a causa de que la pasividad ha permanecido hasta hoy como uno de los conceptos enigmáticos de la investigación husserliana.» (Wälde 1985, p. 69). «[...] que su determinación en los manuscritos publicados hasta el momento se muestra extremadamente insuficiente, breve y formal.» (Holenstein 1972, p. 192). 
constitutiva dadora que opera y es un «presente viviente». Otra de las cuestiones que abordan es la negación de la existencia de una intencionalidad pasiva temporalizadora encargada de la constitución de la temporalidad de la unidad de las vivencias antes de los procesos de captación por parte del yo. Aquí se considera que la intencionalidad pasiva operante en la protoconciencia no es propiamente hablando ninguna intencionalidad ni ejerce ninguna constitución en sentido propio. La constitución activa del yo es la que es constitutiva e introduce la temporalidad. Previamente sólo se puede hablar de una serie de pre-unidades o pre-entes constituidos pasivamente, que necesitan afectar al yo y convertirse en objetos de atención explícita por parte de este para adquirir la categoría de objetos. Ahora bien, que aquí se deje de denominar a esta constitución intencionalidad pasiva no implica que se rechace la necesidad de suponer ciertos procesos constitutivos previos: tiene que presuponerse algún tipo de operación anterior al yo a través de la que se forme una unidad que lo pueda afectar, una fundición protoasociativa [urassoziative Verschmelzung]. Husserl terminará rechazando la idea de que hubiese una cierta intencionalidad pasiva operante en la protocorriente de conciencia, en el sentido de que lo que allí sucede no puede ser denominado intencional. Los manuscritos $\mathrm{C}$ insisten en el carácter activo que tiene la conciencia en la constitución temporal, es decir, se trata más bien de un movimiento de temporalización previo a toda reflexión y que se escapa por definición a esta reflexión, antes que de un flujo estático de vivencias que se suceden el tiempo inmanente. En los manuscritos $\mathrm{C}$ intenta acercarse a aquellas cuestiones no resueltas bajo un nuevo enfoque. Lo cierto es que los cambios se producen más a nivel de la terminología empleada que no a nivel de los contenidos pensados. Este trabajo insiste sobre todo en tres aspectos:

1. La noción de presente viviente apunta a una esfera de validez o donación que no es en sí misma temporal.

En los manuscritos C Husserl descubre que no es suficiente separar pasado y futuro y reducirse al estudio de la dimensión del puro presente. Una reducción más radical que la reducción fenomenológica-trascendental nos lleva a un plano diferente: el presente viviente no es el presente habitual, el presente perceptivo, sino la denominación del movimiento anterior al tiempo inmanente, del movimiento anterior a la entrada en el tiempo; la denominación «presente» se le puede aplicar porque este movimiento es siempre pura actualidad, pero no en el sentido de que sea un intervalo en el tiempo, una duración. Aquí entra en juego un nuevo aspecto de la reflexión motivada por el preguntarse decidido de Husserl acerca de las relaciones entre el yo y el proto-yo, concebido este último como el tener lugar continuo de la temporalización, el presente viviente en el sentido de la presentación viviente, del operar anónimo previo a toda reflexión. El devenir temporal es pensado en su dimensión de causa de que se nos escapen los objetos sobre los que reflexionamos: tiempo como un fluir siempre más allá 
de toda reflexión dirigida hacia él y que en cierto sentido siempre llega tarde. Hasta el último momento, aunque aquí planteado si cabe con mayor claridad, se mantiene el debate entre dos modelos de conciencia: hay una temporalización pasiva que sólo se muestra como tal temporalización a la reflexión o se trata de un operar que ya es temporalización activa previamente a la reflexión dirigida sobre ella. La opción final de Husserl será en estos textos la primera, aunque parece evidente que si hubiese tenido la oportunidad de retomar sus pensamientos no hubiese tenido sin duda dificultades para defender la otra alternativa.

Este protopresente es la esfera de ser actual más originaria a partir de la cual se generan todos los otros tiempos: el tiempo temporalizado de mi yo, el tiempo temporalizado de los otros yos, el tiempo intersubjetivo, etc. Antes que de un presente estático se trata de un movimiento de temporalización o síntesis universal, un movimiento de temporalización en el que se genera todo sentido, es decir, toda dación de sentido es el resultado de esta constitución temporal. El presente viviente es, por lo tanto, cierta estructura anónima que opera, trabaja, constituye, temporaliza de forma continua. Es importante insistir en el carácter no temporal de la noción de presente viviente, en su carácter de actividad.

2. Por último, Husserl apuesta por la negación de la existencia de una intencionalidad pasiva temporalizadora encargada de la constitución de la temporalidad de la unidad de las vivencias antes de los procesos de captación por parte del yo. Aquí se considera que la intencionalidad pasiva operante en la protoconciencia no es propiamente hablando ninguna intencionalidad. El término debe ser reservado para los actos intencionales del yo. Previamente sólo se puede hablar de una serie de pre-unidades o pre-entes constituidos pasivamente, que necesitan afectar al yo y convertirse en objetos de atención explícita por parte de este para adquirir la categoría de objetos, es decir, unidades temporales. Ahora bien, que aquí se deje de denominar a esta constitución intencionalidad pasiva no implica que se rechace la necesidad de suponer ciertos procesos genéticos previos: tiene que presuponerse algún tipo de operación anterior al yo a través de la que se forme una unidad que lo pueda afectar.

3. La reflexión detecta en el operar del presente viviente algo que no sólo la antecede, sino algo que queda más allá de lo que puede traer a dación adecuada: el acto mismo en su operar, la vivencia en su ser vivida. Se «supone» entonces que a la vivencia tal y como es captada inmanentemente en la reflexión tiene que antecederla un operar constitutivo de sentido que ocurre primeramente de forma anónima. Incluso cuando la reflexión que está siendo efectuada reflexiona sobre sí misma esta «estructura» permanece más allá, cerrada sobre sí misma.

En todo caso, se descubre que más que un tiempo lo que caracteriza a la conciencia es un movimiento, un temporalizar. En último extremo es interrogándose acerca de dónde procede este movimiento temporalizador de la conciencia Husserl se ve obligado a analizar el proceso pasivo de donación de las daciones 
hiléticas, es decir, cómo llega la materia (hyle) a la conciencia, como se producen las diferentes donaciones de sentido. Husserl descubre que es la misma la pasividad de la constitución temporal que la pasividad de con la que se configura un mundo en nuestra conciencia. Se descubre una dimensión anónima genética y operante que precede a toda reflexión: es necesario presuponer un tener-vivencia previo sobre el que se realiza la reflexión, una vida operante y un yo anteriores que permanecen anónimos. Tal y como son captados en la reflexión ni los actos ni las unidades hiléticas pertenecen a la corriente de vida [Lebensstrom] que efectúa la prototemporalización sino a la esfera inmanente. Antes de esta hay un movimiento previo pasivo del flujo en el que son vividos como unidades los actos y los datos hiléticos. Se trata de una «conciencia-de» en el sentido de las retenciones y las protenciones que forman parte de este flujo pasivo.

\section{CONCLUSIONES. LA TRANSFORMACIÓN DE LA FENOMENOLOGÍA AL HILO DEL} PROBLEMA DE LA CONCIENCIA DEL TIEMPO

El análisis de Husserl pasa de ser una fenomenología de los objetos temporales a ser una fenomenología de las estructuras de la conciencia que permiten la constitución de esos objetos temporales: la conciencia originaria del tiempo. Se trataba de una denodada lucha por definir que podía ser la retención y encasillarla en alguno de los tipos de actos que había reconocido hasta aquel momento. La retención no podía ser clasificada ni como percepción, ni como conciencia de imagen, fantasía o rememoración, sino que Husserl terminaba por concluir que se trataba de una parte de un acto perceptivo de un tipo peculiar.

Los problemas de la ingrediencia de los contenidos de sensación y del tipo de aprehensión que es necesario para explicar las «aprehensiones» temporales constituían los núcleos iniciales del análisis. El principal descubrimiento es que el esquema que definía la constitución en niveles superiores (la percepción como aprehensión de datos de sensación) no servía para explicar el nivel básico de constitución de la unidad temporal de los datos y de las aprehensiones. La retención desbordaba cualquier clasificación de los actos de conciencia y exigía una explicación totalmente diferente. Los materiales hiléticos se demostraban como algo originario que no era el resultado de ninguna aprehensión previa, sino de otro tipo de constitución. Esto implicaba además el descubrimiento de un plano de conciencia situado por debajo de la conciencia inmanente, dando un paso más allá del análisis inmanente de Ideas I: se trataba de aquello que en un primer momento iba a ser denominado conciencia absoluta y que en la medida en que el estudio se va centrando en su estructura temporal conciencia interna.

El propio concepto de intencionalidad se veía afectado de forma tal que resulta peligroso hablar de una constitución de una intencionalidad constituidora de las cosas. Lo que la fenomenología del tiempo descubre es que las estructuras 
temporales son el resultado de un aparecer directo que no es el resultado de ningún proceso constitutivo en sentido tradicional, sino que implican estructuras mucho más profundas que las estudiadas hasta aquel momento: la conciencia interna. La fenomenología de los objetos temporales, de la conciencia originaria o percepción de objetos que duran, pasa a ser una fenomenología de las vivencias constitutivas de esos objetos, puesto que no sólo las vivencias de objetos que duran, sino toda vivencia dura.

La fenomenología de los años 1907-1909 le permite a Husserl afianzarse en la noción de conciencia absoluta y afirmar que ésta gira en torno a algún tipo de intencionalidad constitutiva diferente de la intencionalidad inmanente constituidora de los objetos que se aparecen. Se descubre que la conciencia absoluta debe estar fuera del tiempo, debe precederlo sin ser ella misma ninguna corriente de contenidos que se suceden en la línea del tiempo. A la vez, Husserl intenta justificar que no es necesario ningún plano de constitución situado por detrás de este, puesto que la conciencia absoluta además de constituir el plano inmanente constituye también sus propias operaciones constitutivas y su unidad. Se trata de una conciencia interna que al mismo tiempo que conciencia de cada una de las vivencias es conciencia de la unidad de estas vivencias y de los peculiares procesos mediante los que se forma la unidad de cada una de éstas. En esta época además reciben su verdadero bautismo los conceptos de protoimpresión, retención y protección, hasta ahora formulados sólo parcialmente o sin una conciencia clara de su contribución o articulación dentro de la conciencia originaria del tiempo.

En los manuscritos de Bernau se presentaban dos concepciones diferentes de lo que es esa conciencia absoluta o conciencia interna, ahora denominada protoproceso: el protoproceso como proceso inconsciente no constitutivo de sucesos (inmanentes) temporales o el protoproceso como constitución temporal activa en todo momento. Otras cuestiones principales debatidas en esta época son una modificación muy importante del concepto de ingrediencia, que lejos de referirse a la presencia «pasiva» o ya constituida, terminada, de un contenido de sensación a la conciencia, pasaba a ser una denominación para el modo originario en que se produce la constitución de determinados contenidos, es decir, no tanto refiriéndose al contenido propiamente dicho, sino a la forma de irrupción de este contenido en la conciencia. Se comprobaba en el estudio de los textos de Bernau que los modelos propuestos se enfrentaban a la objeción de diversos regresos al infinito, pese a lo cual el segundo parecía aquel más probable o de mayor eficacia explicativa. La conciencia era concebida como una conciencia atemporal pero constituidora de temporalidad en todo momento.

Husserl advierte ya en esta época como la investigación deriva por sí misma cada vez más claramente hacia un plano constitutivo previo que precede a toda actividad espontánea del yo, algo que no es lo mismo que afirmar que precede 
a todo lo egoico. Se trata de la génesis pasiva. Más que de una corrección de los análisis anteriores, de lo que se trata aquí es de encarar el análisis desde una perspectiva diferente y más profunda. La protención, la retención y la rememoración son explicadas como fenómenos asociativos y como fenómenos afectivos. La conciencia del tiempo es concebida como una peculiar síntesis de contenidos que viene a rellenar o cumplimentar la estructura de la conciencia formal del tiempo fijada en una época anterior. El rasgo definitorio de sus actividades es que se trata de una intencionalidad pasiva, algo que más bien debería recibir otra denominación, pero que Husserl califica así en virtud de ser aquello que en el plano precedente a las actividades espontáneas le corresponde al operar silencioso de la conciencia, a las síntesis entre retención, protoimpresión y protención.

Los manuscritos C llevan a la fenomenología hasta sus propios límites en la medida en que Husserl descubre que ciertos elementos nucleares sobre los que se levanta la fenomenología van más allá de lo fenomenológico. Se encuentra finalmente con el problema de una pasividad afectiva que es una especie de facticidad última insuperable para el pensamiento. Es decir, por un lado, se trata de una frontera a la reflexión, pero por otro muestra la positividad de un pensamiento que en clara actitud fenomenológica topa y reconoce acertadamente los límites a su capacidad. Si bien es cierto que fenomenología y síntesis pasiva, dación y condiciones de la dación, pueden ser considerados conceptos opuestos (Mishara 1990, p. 53), también lo es que la fenomenología no puede dejar de ser un idealismo «abierto-dialéctico» en virtud de su operar con una serie de conceptos límites que a la par que dentro de una fenomenología estricta injustificables resultan imprescindibles para comprender el ámbito de dación evidente-fenomenológica. La reflexión debe penetrar en aquello prerreflexivo no objetivado, la anonimidad de una ejecución que la precede. Algunos intérpretes demasiado parcialmente le achacan al último pensamiento de Husserl el abandono de los ideales fenomenológicos de descripción exacta de las daciones y afirman que aquí campea la construcción, la especulación y una determinada metafísica. Wälde afirma con rotundidad no sólo que la fenomenología del tiempo es un proyecto destinado desde el principio al fracaso, sino que en su fracasar se lleva por delante también la validez de toda la fenomenología trascendental. Husserl iría progresivamente separándose de la descripción de la realidad de la vida de conciencia para verse atrapado en la construcción de un mundo perceptivo de forma absolutamente teorizante. Quizás sea cierto que en la fenomenología del tiempo las fronteras entre descripción y construcción se hacen más laxas que en otros ámbitos, pero esto no es suficiente para afirmar que aquí se trata de una mera idealización abstractiva (Wälde 1985, p. 33) que se extendería como un cáncer a todo el programa de la fenomenología. 
A nuestro entender, cuando Husserl postula que el yo tiente que ser preafectado por una serie de unidades hiléticas e uniones formadas previamente a la afección en sentido propio, lo hace movido por los resultados de su propia primera fenomenología. Las estructuras descubiertas por la reflexión -el presente viviente-fluyente anónimo- no deben ser atribuidas al ámbito de la pre-reflexividad, sino que son la expresión de la experiencia que la conciencia tiene de sus propios límites.

\section{REFERENCIAS BIBLIOGRÁFICAS:}

\section{Textos Fuente De Husserl}

Hu III: Ideen zu einer reinen Phänomenologie und phänomenologischen Philosophie, Band I, Husserliana, Lovaina, 1950.

Hu X: Zur phänomenologie des inneren Zeitbewußtseins (1893-1917), Husserliana, Band X, Den Haag, Martinus Nijhoff, 1966.

Hu XI: Analysen zur passiven Synthesis. Aus Vorlesungs- und Forschungsmanuskripten 1918-1926, Husserliana, Band XI, Den Haag, Martinus Nijhoff.

Hu XVII: Formale und transzendentale Logik, Husserliana, Band XVII, Martinus Nijhoff, Den Haag, 1974.

Hu XIX/1: Logische Untersuchungen, Husserliana, Lovaina, Band XIX/1, 1984.

Hu XXIV: Einleitung in die Logik und Erkenntnistheorie. Vorlesungen 1906/07, Husserliana, Band XXIV, Martinus Nijhoff, Dordrecht/Boston/Lancaster, 1984.

Hu XXIII: Phantasie, Bildbewußtsein, Erinnerung (1898-1925), Husserliana, Band XXIII, Springer Verlag, 1980.

Hu XXXIII: Die Bernauer Manuskripte über das Zeitbewußtsein (1917/18), Husserliana, Band XXXIII, Springer, Dordrecht, 2000.

Hu - Manuscritos C: Späte Texte über Zeitkonstitution (1929-1934), Die C-Manuskripte, Husserliana Materialien, Tomo 8, Springer, Dordrecht, 2006.

\section{REFERENCIAS SECUNDARIAS:}

DEPRAZ, N. 1994: «Temporalité et affection dans les manuscrits tardifs sur la temporalité (1929-1935) de Husserl», Paris: Alter 2, pp. 63-85.

GARCÍA-BARÓ, M. 1993: La verdad y el tiempo, Salamanca: Sígueme.

HOLENSTEIN. E., 1972: Phänomenologie der Assoziation, La Haya: Phaenomenologica.

MISHARA, R., 1990: «Husserl and Freud: Time, Memory and the Unconsciouss», Husserl Studies 7, Dordrecht, pp. 29-58.

WÄLDE, M. 1985: Husserl und Schapp: Von der Phänomenologie des inneren Zeitbewusstseins zur Philosophie der Geschichte, Basel: Schwabe and Co.

WALTON, R. 1993: Husserl, mundo, conciencia y temporal, Buenos Aires: Almagro. 
Importancia y evolución del concepto de conciencia del tiempo...

Francisco Conde Soto

Universidad de Barcelona

Publicaciones recientes:

«Metafísica de la presencia: La crítica de Derrida en "La voz y el fenómeno" a la teoría del tiempo de Husserl en las "Lecciones sobre la conciencia interna del tiempo"», Paideia: Revista de filosofía y didáctica filosófica, ISSN 0214-7300, Vol. 29, No 82, 2008, pags. 173-192.

«Derrida contra Husserl: la crítica de la voz y el fenómeno a la teoría del signo de la primera investigación lógica de Husserl», en Revista: Investigaciones fenomenológicas, Volumen: 5, pág. 153-207, ed. Sociedad Española de Fenomenología Dpto. de Filosofía y Filosofía Moral y Política (UNED), Madrid, 2007. ISSN: 1137-2400

Líneas de investigación:

Fenomenología, Husserl, Heidegger, postestructuralismo, psicoanálisis.

Dirección postal:

Collège franco-britannique, Chambre 200, 9b Boulevard Jourdan, 75014 Paris

Correo electrónico: francondesoto@yahoo.es 
